


\begin{tabular}{|c|c|c|c|}
\hline$\Omega$ & [1] & $\equiv$ & (2) \\
\hline Binita Kane ${ }^{1,2}$, & 'Manchester Academic & B.R. O'Driscoll: Dept of & ronan.o'driscoll@srft.nhs.uk \\
\hline Samantha & Health Science Centre, & $\begin{array}{l}\text { Respiratory Medicine, } \\
\text { Salford Royal Foundation }\end{array}$ & \\
\hline Decalmer ${ }^{1,2}$, & Dept of Respiratory Medicine, & NHS Trust, Salford M6 & \\
\hline B. Ronan O'Driscoll' & $\begin{array}{l}\text { Salford Royal Foundation } \\
\text { NHS Trust, Salford, UK } \\
{ }^{2} \text { Both authors contributed } \\
\text { equally to this article }\end{array}$ & 8HD, UK & \\
\hline
\end{tabular}

\section{Emergency oxygen therapy: from guideline to implementation}

\section{Educational aims}

- To help readers understand the best way to use oxygen when they care for patients with medical emergencies.

- To encourage best practice in the use of emergency oxygen therapy.

○ To raise awareness of the British Thoracic Society (BTS) guideline for emergency oxygen use.

\section{Summary}

Oxygen is the most commonly used drug in emergency medicine and when used judiciously in the treatment of hypoxaemia it undoubtedly saves life. However, oxygen is often used inappropriately and the dangers of over-oxygenation are unappreciated.

In 2008, the first formal guidance on emergency oxygen use was produced by the British Thoracic Society. The guideline is objective, evidence based and peer reviewed, advocating safe use of oxygen by encouraging target saturation levels to be prescribed for each patient, based on a combination of what is believed to be safe and normal or near-normal. In the majority of patients a target saturation range of $94-98 \%$ is advised. The importance of recognition of patients at risk of type 2 respiratory failure is highlighted and, in such patients, a target saturation range of $88-92 \%$ is recommended.
Statement of Interest B.R. O'Driscoll was paid an honorarium, by the ERS, for delivering a lecture on Emergency Oxygen Therapy at the ERS meeting in Vienna 2009. The BTS has paid his expenses to attend meetings related to the Guideline (no honorarium).

\section{두(1)(으 $\mathrm{ERS}_{2013}$}




\section{Why is a guideline for emergency oxygen necessary?}

\section{Oxygen is the most commonly used drug in emergency medicine}

$34 \%$ of ambulance patients receive oxygen during transit and $15-17 \%$ of hospital inpatients will be receiving oxygen at any given time [1, 2]. Yet prior to 2008 , there was no national or international guidance available for the safe use of oxygen.

\section{There are common misconceptions} regarding the safe use of oxygen and many people are unaware of the dangers of hyperoxaemia

It is widely believed that that supplemental oxygen relieves dyspnoea in the absence of hypoxaemia (low arterial oxygen levels). No evidence of benefit exists for administering oxygen in patients who are normoxaemic (normal arterial oxygen levels) or very mildly hypoxaemic [3, 4]. Dyspnoea can occur for many reasons other than cardiorespiratory disease, including metabolic acidosis, anxiety and pain, and treatment with oxygen is not indicated in these cases.

Another common misconception is that one "can't give too much oxygen" and there is general lack of appreciation for the dangers of "hyperoxaemia" (higher than normal arterial oxygen levels). Historically, high levels of oxygen were given to all patients with dyspnoea and critical illness [5]. It is well established that severe hypoxaemia results in rapid organ failure and death. Oxygen saves lives when used appropriately to correct hypoxaemia and is an essential component in resuscitation of the critically ill; however, there is little evidence that supra-physiological levels of oxygen have a clinical benefit in most instances. Evidence does exist, however, that inappropriate use of oxygen can be detrimental.

Hyperoxaemia can cause coronary vasoconstriction [6]. Paradoxically, therefore, giving too much oxygen at the time of an acute infarction may worsen oxygen delivery to the cardiac muscle. Use of high-flow oxygen has been associated with increased reperfusion injury, infarct size and mortality in myocardial infarction [7, 8]. Theoretically, hyperoxaemia may have similar effects on cerebral blood flow. One randomised controlled trial found that in minor or moderate stroke, oxygen administration was linked to increased mortality when compared with air [9].

High-flow oxygen is commonly used in intensive therapy units (ITU) and hyperoxaemia is common in these wards [10-12]. Studies in critical care have shown that in cardiac arrest survivors $[10,12]$ and in patients receiving ITU care [11, 13], hyperoxaemia is linked to worse outcomes than normoxaemia.

In the ward setting, patients using highflow oxygen without a target saturation range may have high oxygen saturation (>98\%), which can be falsely reassuring to staff. The ability of pulse oximetry to detect clinical deterioration is masked by the high oxygen saturation and patients may become severely hypoxic before the staff are alerted to the deterioration in gas exchange. By contrast, if oxygen administration was titrated against patient need, aiming to achieve a normal oxygen saturation target range, pulse oximetry should allow early detection of increasing oxygen requirements [14, 15].

Inappropriate oxygen use in patients at risk of type 2 respiratory failure (T2RF) can result in life-threatening hypercapnia (higher than normal levels of carbon dioxide in arterial blood), respiratory acidosis, organ dysfunction, coma and death. Vulnerable groups include not only chronic obstructive pulmonary disease (COPD), where high concentrations of inspired oxygen are linked with increased mortality during acute exacerbation [16, 17], but also severe asthma, cystic fibrosis, bronchiectasis, chest wall disorders, neuromuscular disease and obesity hypoventilation [5, 18]. All at-risk patients need to be identified when prescribing and administering oxygen.

In 2010, the UK National Patient Safety Agency (NPSA) [19] reported nine deaths directly attributable to oxygen therapy over a 5-year period. The four reported deaths due to over-oxygenation are thought to be a gross underestimation as previous work in COPD has estimated that, in the UK, a few thousand deaths could be avoided each year by controlled oxygen use $[16,17,20,21]$. The NPSA relies on clinicians reporting adverse events.

\section{Oxygen prescribing is poor}

Despite being a drug, oxygen is often not prescribed appropriately, signed for on drug 
charts or regularly reviewed. The 2008 national oxygen audit (carried out prior to the publication of the emergency oxygen guideline) showed that, in UK hospitals, less than onethird of patients receiving oxygen had any written prescription, in only $10 \%$ of cases was consideration given to a target saturation range and in only $5 \%$ of cases was oxygen signed for to indicate it had been administered [2].

The British Thoracic Society (BTS) Emergency Oxygen Guideline, published in 2008 , addresses many of the issues surrounding the use and prescribing of oxygen, specifically regarding target saturation ranges [5]. The recommendations aim to guide clinicians, encouraging levels of oxygenation that are appropriate for each patient, based on a combination of what is believed to be safe and normal or near-normal.

\section{How was the guideline produced?}

A working party was established by the BTS Standards of Care Committee in 2003. A bespoke literature search identified 3306 papers and, following abstract review, 184 relevant articles were evaluated in their entirety. In conjunction with representatives from a number of societies and colleges with an interest in emergency oxygen (21 in total), objective, evidence based, peer reviewed guidelines were produced in 2008, aimed at all healthcare professionals using oxygen and endorsed by a number of professional bodies including Royal College of Physicians, Resusitation Council (UK), Society of Emergency Medicine, Intensive Care Society, Royal College of Anaesthetists and
British Paramedic Association, to name but a few [5].

\section{Key messages from the guidelines}

The guidelines cover the use of oxygen in critically ill and hypoxaemic adults and those who are at risk of hypoxaemia. A number of central points are addressed.

\section{Oxygen is a treatment for hypoxaemia}

As mentioned above, there is little evidence for the use of supplemental oxygen in the non-hypoxaemic patient (exceptions to the rule include treatment of carbon monoxide poisoning and pneumothorax).

\section{Oxygen is an essential part of resuscitation and patient stabilisation in critical illness}

Pulse oximetry should be recorded in all patients as the 'fifth vital sign' and further assessment with arterial blood gases (ABGs) performed, if indicated (see box).

All patients with severe hypoxaemia (including arrest and peri-arrest situations), acute breathlessness, severe sepsis and any other critical illness should be given highconcentration supplemental oxygen in the initial stages of the resuscitation process. Once the patient is stable, formal assessment of the need for oxygen should be made, guided by pulse oximetry plus $A B G s$ if required.

Arterial blood gases (ABGs) are recommended in:

- All critically ill patients

- Unexpected/inappropriate hypoxaemia (oxygen saturation $<94 \%$ or requiring supplemental oxygen to maintain this)

- Deteriorating saturation, increasing oxygen need or increasing dyspnoea in a previously stable patient

- Patients at risk of type 2 respiratory failure with acute dyspnoea, decreasing saturation, drowsiness or other symptoms suggestive of hypercapnia.

- Dyspnoeic patients who are at risk of metabolic acidosis (e.g. diabetes and renal failure)

- Dyspnoeic or critically ill patients with an inadequate/unreliable oximetry signal due to poor peripheral circulation.

- Unexpected change in early warning score or fall of $>3 \%$ in saturation 
In all acutely unwell patients, not at risk of $\mathrm{T} 2 \mathrm{RF}$, the recommended target saturation range is $94-98 \%$

Evidence suggests that in healthy nonsmoking adults, saturations of $96-98 \%$ are normal. Oxygen saturation diminishes slightly with age and in patients over the age of 70 years saturation of $<94 \%$ may be normal for a particular patient, especially if there is underlying lung disease or heart failure. If the patient is clinically stable, supplemental oxygen is not required $[22,23]$.

Research from intensive care medicine shows impaired median-term survival with oxygen saturation $<90 \%$; therefore, in critical illness, it is recommended that saturation be kept at $>90 \%$ [24-28].

As most ward settings rely on pulse oximetry to measure oxygenation rather than the invasive monitoring that occurs in critical care areas, the BTS recommends a 'normal' target saturation of $94-98 \%$. This allows a margin of safety (90-94\%) for possible inaccuracies in pulse oximetry readings and a "safety cushion" to allow for fluctuation in the oxygen saturation.

In patients at risk of $\mathrm{T} 2 \mathrm{RF}$ a target saturation range of $88-92 \%$ is suggested pending the availability of ABGs

This is based on results from research, primarily in COPD. First, in acute exacerbations of COPD, achieving oxygen saturation greater than $85 \%$ has been shown to prevent death [17]. Secondly, nearly half of patients with acute exacerbation of COPD have hypercapnia. The majority of hypercapnic patients with arterial oxygen levels in excess of $10 \mathrm{kPa}$ (equivalent to a saturation of about 92-93\%) have associated acidosis [17]. Hence, a target range of $88-92 \%$ is recommended and this has been extrapolated from COPD to include other groups at risk of T2RF. The lower limit is set at $88 \%$ rather than $85 \%$ to allow for potential inaccuracies in pulse oximeter readings.

Not all individuals with COPD will develop $\mathrm{T}_{2} \mathrm{RF}$ with oxygen therapy. If there is no evidence of hypercapnia on ABGs, target saturation should be increased to $94-98 \%$, providing there are no previous episodes of $T_{2} R F$ or requirement for non-invasive or invasive ventilation. Any alteration in oxygen therapy should be reassessed in 30-60 min by repeat ABC.
If there is no known or documented evidence of COPD, but a presenting patient is $>50$ years old and a long-term smoker with a history of dyspnoea on exertion it is recommended they are treated as COPD until ABGs are available.

Oxygen is a drug and hence should be prescribed, administered and monitored by trained staff

In life-threatening situations, high-flow oxygen via a reservoir (non-rebreathe) bag should be given immediately, without a prescription, but subsequent documentation should take place.

In all other situations, oxygen should be prescribed by a doctor, on a designated document (usually the drug chart) and signed for at each drug round by trained staff.

Guidelines advocate that oxygen is prescribed with a target saturation range, initial delivery device and flow rate and is regularly reviewed by oximetry. Increasing oxygen requirement, decreasing saturation or increasing respiratory rate may herald patient deterioration and should prompt rapid medical assessment. As oxygen requirements decrease, supplemental oxygen can be titrated downwards and eventually discontinued, but the prescription for an oxygen target range should remain active in case the patient deteriorates again.

In conditions where there is risk of $T_{2} R F$, Venturi masks are the delivery device of choice as constant or known oxygen concentrations are administered, regardless of flow.

In most breathless but non-hypoxaemic patients, supplemental oxygen is not required

However, if a patient's oxygen saturation falls by $\geqslant 3 \%$, even if still within the target range, prompt assessment should ensue (including validity of the pulse oximetry trace) as it may herald acute deterioration in the patient's condition.

If patients carry an oxygen alert card, due to previous T2RF, the patient specific target range should be prescribed

Patients who have had previous episodes of T2RF should be issued with an oxygen alert card (documenting an appropriate target 
saturation range) and a Venturi mask (either $24 \%$ or $28 \%$ ) so that treatment in subsequent emergency is tailored to patient requirements.

The BTS recommendations for oxygen use in a number of clinical situations are shown in Table 1.

\section{How was the guideline implemented?}

Copies of the guidelines were sent to all hospital chief executives, medical directors, nursing heads and to all primary care and ambulance trusts, as well as to education leads in medical and nursing schools.

Local oxygen champions were identified to review local oxygen policy in accordance with the national guidelines, arrange staff education, and ensure oxygen prescription and monitoring could be achieved on drug and observation charts. Lectures, teaching material and example documentation were made available through the BTS website. Local oxygen champions were also instrumental in re-auditing after the initial implementation of the policy.

\section{The impact of the guidelines on clinical practice: key results from the BTS oxygen audits}

BTS emergency oxygen audits [2] are performed annually and, since the guidelines were implemented in 2008, the number of participating trusts has increased from 47 in 2009 to 145 in 2012, with the most recent audit encompassing 1733 hospital wards and 38094 patients. The uptake of the guidelines has been excellent, with $83 \%$ of participating trusts having implemented an oxygen policy by 2012 , compared with $6 \%$ in 2009 .

The audit results show that oxygen use and prescribing are improving, albeit slowly. In 2008, $32 \%$ of patients who were using oxygen had some form of prescription; this had risen to $69 \%$ in 2009 . By 2012, the figure had dropped to $52 \%$, but this may be largely due to selection bias rather than a true drop in prescribing levels. Despite the improvements, disappointingly nearly half of all oxygen in use still remains unprescribed.

Table 1. Recommendations for emergency oxygen use

1. Critical illness requiring high levels of supplemental oxygen
Give $15 \mathrm{~L} \cdot \mathrm{min}^{-1}$ via a reservoir mask and once stable, reduce oxygen to aim for a saturation range of $94-98 \%$.

If patient at risk of $\mathrm{T}_{2} \mathrm{RF}$, aim for the same initial saturation as all other critically ill groups pending $A B G$.

Initially give 2-6 L· $\mathrm{min}^{-1}$ via nasal cannulae or 5-10 L· $\mathrm{min}^{-1}$ via facemask, aiming for a saturation range of $94-98 \%$. If saturation can't be maintained or initial saturation is $<85 \%$, use a reservoir mask with $10-15 \mathrm{~L} \cdot \mathrm{min}^{-1}$.

If at risk of $T_{2} R F$, aim for saturation of $88-92 \%$, adjusting to $94-98 \%$ if the $A B G$ s show normal carbon dioxide ${ }^{\#}$. Repeat ABG after $30-60$ min.

Before $A B C$ use a $28 \%$ Venturi mask $\left(4 \mathrm{~L} \cdot \mathrm{min}^{-1}\right)$, aiming for a saturation range of 88 $92 \%$, adjusting to $94-98 \%$ if the $A B G$ s show normal carbon dioxide ${ }^{\#}$. Repeat $A B G$ after 30-60 min.

If the patient has an oxygen alert card, aim for the target range specific to him/her. If the patient is hypercapnic and acidotic, despite $30 \mathrm{~min}$ of appropriate treatment and oxygenation, consider non-invasive ventilation.

If hypoxaemia develops, follow recommendations as per serious illness (point 2 above)

4. Conditions for which the patient should be closely monitored but oxygen is not required unless hypoxic

\footnotetext{
\#: Unless there is a history of previous hypercapnic respiratory failure requiring non-invasive or invasive ventilation, in which case the target saturation should remain at 88-92\%; ": If no diagnosis is known but the patient is $>50$ years old and a long-term smoker with chronic dyspnoea, treat as presumed COPD. Adapted from the British Thoracic Society guideline for emergency oxygen use in adult patients (tables 1-4) [5].
} 


\section{Educational questions}

1. Which of the

following statements

are true?

a. The

recommended target

saturation range for

patients not at risk of

T2RF is $92-94 \%$.

b. For most patients

with COPD, target

saturation range

should be set at 88-

$92 \%$ until blood gases

are available.

c. For all critically ill patients, high

concentration oxygen

should be

administered

immediately until the

patient is stable.

d. Only patients

with COPD are at risk

of $T_{2} R F$.

2. Which of the

following are true?

a. Oxygen is

indicated for all

breathless patients.

b. Oxygen is

indicated in a patient with saturation $98 \%$

on room air.

c. Oxygen is

indicated in a patient who is suffering an acute $\mathrm{MI}$ who has saturation of $90 \%$.

d. Oxygen should be given to all patients having an acute stroke regardless of oxygen saturation.

3. A patient with COPD and a history of hypercapnic respiratory failure becomes very breathless on the ward. On arrival his
The prescription of target saturation ranges has improved, from $10 \%$ to $46 \%$. Overall, the proportion of hospital inpatients using oxygen has reduced from $17.5 \%$ in 2008 to $14 \%$ in 2012 . The percentage of patients in UK hospitals using oxygen with no written order has reduced from $11.9 \%$ in 2008 to $6.7 \%$ in 2012 , which is encouraging.

There is still much work to be done before the guidelines are being adhered to universally. The major challenge remains the training of healthcare professionals, which is a main aim for the BTS emergency oxygen guidelines committee.

\section{Key new publications on oxygen since 2008}

The dangers of hypoxaemia are well documented, the most recently published studies on oxygen therapy focus on the dangers of hyperoxaemia which is a more controversial area, with many clinicians still believing that "more is better".

Three large studies have been performed in critical care settings which have produced conflicting results. DE JONGE et al. [13] performed a retrospective observational study on oxygenation in the first $24 \mathrm{~h}$ after admission to the ITU in 36307 consecutive patients who were mechanically ventilated. The results showed that in-hospital mortality was increased with both abnormally low and abnormally high oxygen levels. However, multicentre observational data from Australia (12 108 patients) looking at the worst $A B G$ in first $24 \mathrm{~h}$ following admission, suggested that supranormal oxygen levels did not predict hospital mortality after multivariate analysis [11], although this study confirmed the finding of a higher mortality rate in hypoxaemic patients.

The third study was an observational study, based on a database of 6326 adult patients from 120 ITUs admitted after resuscitation from cardiac arrest. This study showed that arterial hyperoxaemia was independently associated with increased in-hospital mortality compared with either hypoxaemia or normoxaemia [12] and that there was a dose-dependent association between supranormal oxygen levels and the risk of inhospital death [29].

These findings should be interpreted with some caution as they are observational and retrospective and also because the relationship between oxygen and mortality is not necessarily causal. It is, therefore, difficult to draw sound conclusions from these studies, but it is reasonable to recommend that keeping oxygen saturation levels in the normal range (94$98 \%$ ) in critically ill patients is best, as no increase in mortality was seen in the normoxaemic range in all three studies.

Other studies have investigated use of oxygen in emergency care settings. The dangers of high-flow oxygen in COPD have been well documented since the 1960s [17]. However, the first randomised trial of controlled oxygen therapy in acute exacerbations of COPD was published in 2010 and confirmed that mortality was increased when high concentration oxygen was given compared with controlled oxygen, with a target range of $88-92 \%$, (mortality 9\% versus 4\%, respectively) [20]. Patients given controlled oxygen were also much less likely to develop respiratory acidosis or hypercapnia. Similarly, the risk of hypercapnia in obesity hyperventilation syndrome is well established; however, the first randomised placebo-controlled trial in obesity hyperventilation syndrome was published in 2011 and showed that breathing $100 \%$ oxygen worsened hypercapnia in stable patients with obesity hyperventilation syndrome [30].

Recent randomised controlled trials have shown that using high concentration oxygen is associated with increased risk of hypercapnia in acute asthma and pneumonia [31, 32], conditions where the risk of hypercapnia is perhaps less well recognised. Patients were randomised to receive either high concentration oxygen ( $8 \mathrm{~L} \cdot \mathrm{min}^{-1}$ via simple facemask) or titrated oxygen to achieve a target saturation of $93-95 \%$ for 60 min. In acute asthma, all 10 cases of hypercapnia recorded occurred among the patients given high concentration oxygen. In pneumonia, there was a higher proportion of patients with a $>4 \mathrm{mmHg}$ rise in carbon dioxide in the high oxygen concentration group $(57.1 \%$ versus $12.8 \%$ ), which was statistically significant. These are the first randomised controlled studies to suggest that increases in carbon dioxide in response to high concentration oxygen are not just limited to COPD and other diseases with a well-recognised risk of hypercapnia.

As described earlier, studies have suggested that high-flow oxygen can be detrimental in acute myocardial infarction, but 
this is contradicted by the recent OPTIMISE study [33]. This was a pilot study, in which 163 patients presenting with ST elevation myocardial infarction (STEMI) were randomised to either high-flow oxygen $\left(6 \mathrm{~L} \cdot \mathrm{min}^{-1}\right.$ via facemask) or controlled oxygen with target saturation of $94-98 \%$ prior to emergency percutaneous coronary intervention $(\mathrm{PCl})$. No difference was found between the two arms of the study in 30 day mortality or infarct size. This study was flawed in that patients were randomised to treatment in hospital and most had received high-flow oxygen in the ambulance en route to hospital. Therefore, the results only apply to the short period of time between admission to hospital and primary $\mathrm{PCl}$. The ongoing Air Versus Oxygen In myocarDial infarction (AVOID) study is a multicentre randomised controlled trial comparing high-flow versus controlled oxygen in STEMI. This study will enrol 490 patients and includes controlled oxygen therapy in the prehospital setting [34]. We look forward to the publication of the results, which may provide some clarity for the optimal use of oxygen in acute myocardial infarction.

\section{Updating the current guideline}

The next version of the BTS emergency oxygen guideline will be titled: "BTS guideline for oxygen use in adults in healthcare and emergency settings" and there will be a separate guideline on emergency use in children. The key principles will remain that oxygen is a treatment of hypoxemia and that oxygen should be prescribed to a target range. The target ranges specified in the 2008 guideline are likely to remain unchanged. The new children's guideline will provide comprehensive guidance on the emergency use of oxygen in paediatric healthcare and the adult guideline has been extended to include first responders and palliative care settings. Use of oxygen in continuous positive airway pressure ventilation systems, heliox and nitrous oxide mixtures, procedures that require conscious sedation, the peri-operative period and in track and trigger warning systems (e.g. early warning scores) will also be included. Publication is anticipated in 2014.

Key points

- Oxygen is a treatment for hypoxaemia not breathlessness.

- Oxygen is a drug and should be prescribed with a target saturation range.

- The recommended oxygen target saturation range in patients not at risk of type II respiratory failure is $94-98 \%$.

- The recommended oxygen target saturation range in patients at risk of type II respiratory failure is $88-92 \%$.

\section{References}

1. Hale KE, Gavin C, O'Driscoll BR. Audit of oxygen use in emergency ambulances and in a hospital emergency department. Emerg Med J 2008; 25: 773-776.

2. O'Driscoll BR, Howard LS, Bucknall C, et al. British Thoracic Society emergency oxygen audits. Thorax 2011; 66: 734-735.

3. O'Driscoll BR. Short burst oxygen therapy in patients with COPD. Monaldi Arch Chest Dis 2008; 69: 70-74.

4. Abernethy AP, McDonald CF, Frith PA, et al. Effect of palliative oxygen versus room air in relief of breathlessness in patients with refractory dyspnoea: a double-blind, randomised controlled trial. Lancet 2010; 376: 784-793.

5. O'Driscoll BR, Howard LS, Davison AG. BTS guideline for emergency oxygen use in adult patients. Thorax 2008; 63: Suppl. 6, vi1-vi68.
6. McNulty PH, King N, Scott S, et al. Effects of supplemental oxygen administration on coronary blood flow in patients undergoing cardiac catheterization. Am J Physiol Heart Circ Physiol 2005; 288: $\mathrm{H}_{1057-\mathrm{H}_{106} \text {. }}$

7. Farquhar $\mathrm{H}$, Weatherall $\mathrm{M}$, Wijesinghe $\mathrm{M}$, et al. Systematic review of studies of the effect of hyperoxia on coronary blood flow. Am Heart J 2009; 158: 371-377.

8. Kaneda $\mathrm{T}, \mathrm{Ku} \mathrm{K}$, Inoue $\mathrm{T}$, et al. Postischemic reperfusion injury can be attenuated by oxygen tension control. Jpn Circ J 2001; 65: 213-218.

9. Rønning OM, Guldvog B. Should stroke victims routinely receive supplemental oxygen? A quasirandomized controlled trial. Stroke 1999; 30: 2033-2037. oxygen saturation is $82 \%$ on room air, the correct course of action is:

a. Start immediately on high flow oxygen.

b. Do not give oxygen until blood gas results are available.

c. Start 24 or $28 \%$ oxygen via a Venturi mask, then check blood gases.

d. Do nothing, he is known to have COPD and is often breathless and anxious.

4. Which of the following statements regarding oxygen prescribing are true?

a. Oxygen is a drug and should be prescribed.

b. In life-threatening emergencies, oxygen can be given without a prescription until the patient is stable.

c. Oxygen does not need to be signed for on a drug chart.

d. If a patient's oxygen requirements increase, medical assessment is needed. 


\section{Suggested answers}

1. b, c.
2. c.
3. c.
4. a, b, d.

10. Bellomo R, Bailey M, Eastwood GM, et al. Arterial hyperoxia and in-hospital mortality after resuscitation from cardiac arrest. Crit Care 2011; 15: Rgo.

11. Eastwood G, Bellomo R, Bailey M, et al. Arterial oxygen tension and mortality in mechanically ventilated patients. Intensive Care Med 2012; 38: 91-98.

12. Kilgannon JH, Jone $\mathrm{AE}$, Shapiro NI, et al. Association between arterial hyperoxia following resuscitation from cardiac arrest and in-hospital mortality. JAMA 2010; 303: 2165-2171.

13. de Jonge $E$, Peelen $L$, Keijzers $P$, et al. Association between administered oxygen, arterial partial oxygen pressure and mortality in mechanically ventilated intensive care unit patients. Crit Care 2008; 12: R156.

14. Downs JB. Has oxygen administration delayed appropriate respiratory care? Fallacies regarding oxygen therapy. Respir Care 2003; 48: 611-620.

15. Thomson AJ, Webb DJ, Maxwell SR, et al. Oxygen therapy in acute medical care. BMJ 2002; 324: 1406-1407.

16. Roberts CM, Stone RA, Buckingham RJ, et al. Acidosis, non-invasive ventilation and mortality in hospitalised COPD exacerbations. Thorax 2011; 66: 43-48.

17. Murphy R, Driscoll P, O'Driscoll R. Emergency oxygen therapy for the COPD patient. Emerg Med J 2001; 18: 333-339.

18. British Thoracic Society Scottish Intercollegiate Guidelines Network. British Guideline on the management of asthma. Thorax 2008; 63: Suppl. 4, iv1-iv121.

19. National Patient Safety Agency. Frequently Asked Questions. Implementing the Rapid Response Report 'Oxygen Safety in Hospitals'. National Patient Safety Agency, 2009. Available from: www.nrls.npsa. nhs.uk/resources/?entryid $45=62811$ Date last updated: March 29, 2010; Date last accessed: May 17, 2013.

20. Austin MA, Wills KE, Blizzard L, et al. Effect of high flow oxygen on mortality in chronic obstructive pulmonary disease patients in prehospital setting: randomised controlled trial. BMJ 2010; 341: 05462 .

21. Price LC, Lowe D, Hosker HS, et al. UK national COPD audit 2003: impact of hospital resources and organisation of care on patient outcome following admission for acute COPD exacerbation. Thorax 2006; 61: 837-842.

22. Crapo RO, Jensen RL, Hegewald M, et al. Arterial blood gas reference values for sea level and an altitude of 1,400 meters. Am J Respir Crit Care Med 1999; 160: 1525-1531.
23. Witting MD, Scharf SM. Diagnostic room-air pulse oximetry: effects of smoking, race, and sex. Am J Emerg Med 2008; 26: 131-136.

24. Brain Trauma Foundation, American Association of Neurological Surgeons, Congress of Neurological Surgeons. Guidelines for the management of severe traumatic brain injury. J Neurotrauma 2007; 24: Suppl. 1, S1-S106.

25. Slutsky AS. Consensus conference on mechanical ventilation - January 28-30, 1993 at Northbrook, Illinois, USA. Part I. European Society of Intensive Care Medicine, the ACCP and the SCCM. Intensive Care Med 1994; 20: 64-79.

26. Dellinger RP, Carlet JM, Masur $\mathrm{H}$, et al. Surviving Sepsis Campaign guidelines for management of severe sepsis and septic shock. Crit Care Med 2004; 32: 858-873.

27. Bowton DL, Scuderi PE, Haponik EF. The incidence and effect on outcome of hypoxemia in hospitalized medical patients. Am J Med 1994; 97: 38-46.

28. Jubran A, Tobin MJ. Reliability of pulse oximetry in titrating supplemental oxygen therapy in ventilatordependent patients. Chest 1990; 97: 1420-1425.

29. Kilgannon JH, Jones AE, Parrillo JE, et al. Relationship between supranormal oxygen tension and outcome after resuscitation from cardiac arrest. Circulation 2011; 123: 2717-2722.

30. Wijesinghe M, Williams M, Perrin K, et al. The effect of supplemental oxygen on hypercapnia in subjects with obesity-associated hypoventilation: a randomized, crossover, clinical study. Chest 2011; 139: 1018-1024

31. Perrin K, Wijesinghe M, Healy $\mathrm{B}$, et al. Randomised controlled trial of high concentration versus titrated oxygen therapy in severe exacerbations of asthma. Thorax 2011; 66: 937-941.

32. Wijesinghe M, Perrin K, Healy B, et al. Randomized controlled trial of high concentration oxygen in suspected community-acquired pneumonia. J $R$ Soc Med 2012; 105: 208-216.

33. Ranchord AM, Argyle R, Beynon R, et al. Highconcentration versus titrated oxygen therapy in STelevation myocardial infarction: a pilot randomized controlled trial. Am Heart J 2012; 163: 168-175.

34. Stub D, Smith K, Bernard S, et al. A randomized controlled trial of oxygen therapy in acute myocardial infarction Air Verses Oxygen In myocarDial infarction study (AVOID Study). Am Heart J 2012; 163: 339-345. 